\title{
Weight loss, program completion and biometric changes by location of a healthy living program
}

\begin{abstract}
Objectives: The Healthy Living Program (HLP) is a behavioral change intervention which utilizes group support, health education and organized group exercise to promote healthier dietary practices and physical activity in urban underserved areas of Rochester, NY. Over 200 HLP groups have been completed at 20 different locations since 2001. Location types include Faith Centers, Community Centers and Health Centers. New models from the implementation literature recommend considering the program location and staff equally important as the intervention itself. Our objective is to see if there is a relationship between the type of location of a behavioral change intervention and program completion, weight loss and biometric measurement change.
\end{abstract}

Study design: This is a secondary analysis of data looking at differences in outcomes based on the type of location of the intervention. The primary outcome is weight loss, using both absolute weight loss in pounds and categorical weight loss percent. We also analyzed change in systolic and diastolic blood pressures, waist and hip circumference and program completion based on type of location.

Methods: The first analysis was restricted to only those cases with a full set of values and then a second analysis was completed using imputed data with an intention to treat approach. Differences in baseline characteristics between the groups were analyzed using the Pearson chi-square test. For continuous variables significant differences were determined using repeated-measures analysis of variance. Percentages of participants in each group who met different categorical weight losses $(<5 \%$ or $\geq 5 \%)$ were compared using the Pearson chi-square test. Completion of at least one variable of the post-assessment was considered "Program Completion", and analyzed using the chi-square test. We used generalized linear modeling to assess differences in changes related to gender, age and race/ethnicity, baseline BMI and group class size.

Results: There was a small, but significant difference in the weight loss and percentage weight loss of those who participated in a Health Center based HLP compared with participants in Faith and Community Center based HLPs. We found the highest rates of program completion in Faith Based Center HLPs. There were no differences in changes in blood pressure or waist or hip circumference changes.

Conclusions: This study provides preliminary evidence that type of location may be an important element when designing and implementing interventions and that health center based interventions should be a focus of further obesity treatment research.
Volume 7 Issue 2 - 2017

\author{
Holly Ann Russell,' Colleen T Fogarty,' \\ Nancy M Bennett, ${ }^{2}$ Jennifer Carroll,' Kevin \\ Fiscella,' Ann Dozier ${ }^{3}$ \\ 'Department of Family Medicine, University of Rochester \\ School of Medicine and Dentistry, USA \\ ${ }^{2}$ Center for Community Health, University of Rochester School \\ of Medicine and Dentistry, USA \\ ${ }^{3}$ Department of Public Health Sciences, University of Rochester \\ School of Medicine and Dentistry, USA
}

\begin{abstract}
Correspondence: Holly Ann Russell, University of Rochester School of Medicine and Dentistry, Department of Family Medicine, 777 South Clinton Avenue, Rochester, NY, USA, 14620, Tel 5852794600, Fax 5852794605,

Email Holly_Russell@urmc.rochester.edu
\end{abstract}

Received: July 17, 2017 | Published: July 26, 2017

Keywords: program evaluation, obesity, health behavior

\section{Introduction}

The prevalence of obesity among adults in the United States has increased from $12 \%$ in 1991 to $33.8 \%$ in $2011 .{ }^{1}$ More than two thirds of the adult population is overweight or obese and $25 \%$ engage in no leisure time physical activity. ${ }^{1}$ While the economic and public health costs of obesity and inactivity are far reaching, many of the effects can be reversed. A recent review of lifestyle modification for obesity/ overweight identified a comprehensive approach including caloric restriction, physical activity and behavior therapy as the first line approach for weight loss. ${ }^{2}$

The Healthy Living Program (HLP) is a 12-week, 24-session program which combines physical activity and nutrition instruction delivered to an underserved, at-risk adult population in Rochester, NY at 14 sites, including churches, health centers and community centers. Designed as a behavior change intervention, the HLP promotes healthy lifestyle change, rather than specifically weight loss.
However, the goal of many participants is weight loss. This project builds on our qualitative research regarding barriers and facilitators to behavioral change using focus group narratives from participants in a health center based HLP. Many participants indicated that the location of the intervention, involvement of their health-care providers and familiarity with program staff were facilitators for their successful lifestyle changes. "I liked Dr C, she was not my regular doctor, but she knows me. She knows some of my issues and stuff like that. That is what I like about coming here, my health issues are known, they knew what was wrong with me. Cheryl knew about the high blood pressure, the pre-diabetes, Dr C too".3

While several characteristics associated with successful behavioral change interventions are clear, many questions remain unanswered, including optimal ages to begin obesity interventions, methods for implementing effective clinical trials on a larger scale in real communities, and predictors of success in obesity interventions among different populations. ${ }^{4}$ An emerging model from the field of 
implementation science recommends a contextual view recognizing interrelated systems as opposed to a "mechanistic, determinism approach to science". ${ }^{5}$ In this model, the program delivery site and staff are deemed as important as the intervention and research team.

The RE-AIM (Reach, Efficacy, Adoption, Implementation and Maintenance) framework was developed to help evaluate the public health impact of health promotion interventions. This framework recognizes that traditional interventions tend to be studied using highly standardized protocols in controlled study centers and result in expensive programs that may not work in busy Primary Care Physician (PCP) offices, community or faith centers. The authors describe a "flaw" in the basic design of interventions that the "characteristics that make an intervention efficacious (e.g. level of intensity of the intervention and whether it is designed for motivated, homogeneous populations) work against its being effective in more complex, less advantageous settings with less motivated patients and overworked staff'. ${ }^{6}$ Multilevel Interventions understand the role of context, but even in this field, a review found that context is not fully reported and they are frequently lacking descriptions of how to implement these types of interventions. ${ }^{7}$

Viewed through this contextual lens, it becomes clear that an intervention's location may be more important than previously considered. Most obesity research has focused on the intervention itself rather than the program delivery site. ${ }^{8}$ Given the complexity of addressing this epidemic it is important to compare the locations of the interventions to see what advantage, if any, location type may have. This type of comparison has not been done previously, but the information could be valuable and inform modifications in the implementation and dissemination of the HLP and future behavioral change interventions.

\section{Methods}

\section{Program}

The Healthy Living Program (HLP) is a behavioral change intervention which utilizes group support, health education and organized group exercise. The HLP developed by a community coalition and staffed by the University of Rochester Medical Center's Center for Community Health (URMC-CCH), promotes healthier dietary practices and physical activity in urban underserved areas of Rochester. The curriculum was adapted in part from the Stanford University Patient Education Research Center's living a Healthy Life with a Chronic Condition ${ }^{9}$ and modified for a local audience with input from a community coalition that included members of a variety of community organizations as well as members of the target communities.

The HLP consists of 24 bi-weekly 90 minute group sessions. Each session's core elements include a 60minute physical activity program and a 30minute health promotion/behavioral change interactive curriculum addressing nutrition, preventive health services, stress reduction and smoking cessation. The HLP maintains a unique focus on lifestyle changes and personal goals rather than weight loss as measure of success. The sessions are held in three different type of locations; Faith Centers, Health Centers and Community Centers.

\section{Study population}

Each HLP course enrolls approximately 25 participants over 18 and able to engage in a physical activity program. Participants are referred to the health center based HLPs by their Primary Care Physicians and to faith and community-center based HLPs through flyers, bulletins and announcements. The Physical Activity Readiness Questionnaire
(PAR-Q) is used to screen participants prior to enrollment, with medical clearance requested as necessary. From January 2008 through March 2012, 566 first-time participants enrolled in the HLP. Of the 566 participants, $212(37.5 \%)$ participated in HLP groups at faith centers or churches, $131(23.1 \%)$ participated at health centers and $223(39.4 \%)$ participated at community centers. This analysis of deidentified data was deemed exempt after review by the University of Rochester Medical Center Research Subjects Review Board.

\section{Measures}

At the first session of each HLP course, participants complete a 32-item self-administered questionnaire developed by the Center for Community Health including demographic, health history and health behavior information. Baseline biometric measures, including height, weight, blood pressure, resting heart rate, waist and hip circumference were assessed by a certified fitness trainer. The questionnaire and measurements were repeated at the end of the 12-week program.

\section{Biometric outcomes}

The primary outcome of interest for the analysis was weight loss, using both absolute weight loss in pounds and categorical weight loss percent. We chose a cut off of 5\% because weight losses of 5-10\% are associated with clinically relevant improvements in CVD risk factors such as blood pressure, $\mathrm{HbA1C}$ and triglycerides. ${ }^{10} \mathrm{We}$ also analyzed change in systolic and diastolic blood pressures. In a large meta-analysis, reductions in systolic blood pressure of $7.5 \mathrm{mmHg}$ and diastolic blood pressure of $4.5 \mathrm{mmHg}$ were associated with a $24 \%$ risk reduction in stroke and $13 \%$ risk reduction in myocardial infarction. ${ }^{11}$ We analyzed change in waist and hip circumference. Reductions in waist circumference are also associated with significant improvements in cardiovascular risk factors. ${ }^{12}$ We elected not to use body mass index (BMI) as a separate variable as waist circumference is considered a better predictor of obesity-related health risk than BMI. ${ }^{13}$

\section{Data analysis}

We analyzed the data first assuming that the probability of missing data on one variable was not related to the value of that variable or to other variables in the data set (missing completely at random) and restricted our analysis to those cases with a full set of values. For this analysis, we analyzed only those participants who completed both a pre- and post-assessment for a given variable. For the second analysis we imputed data using an intention to treat approach by carrying forward the value of the last observation for any missing data.

Differences in baseline characteristics between the groups were analyzed using the Pearson chi-square test. For continuous variables significant differences were determined using repeated-measures analysis of variance. Percentages of participants in each group who met different categorical weight losses $(<5 \%$ or $\geq 5 \%)$ were compared using the Pearson chi-square test. Completion of at least one variable of the post-assessment was considered "Program Completion", and analyzed using the chi-square test. We used generalized linear modeling to assess differences in changes related to gender, age and race/ethnicity, baseline BMI and group class size. ${ }^{14}$ We considered a $\mathrm{p}$ value less than 0.05 a significant difference. All analyses were conducted using SAS version 9.2.

\section{Results}

\section{Demographic characteristics}

There were significant between-group baseline differences in age, weight, height, systolic blood pressure, hip and waist circumference, class size, and race (Table 1). 
Table I Baseline descriptive summary

\begin{tabular}{|c|c|c|c|c|c|}
\hline & Total & Faith centers & Health centers & Community centers & $P$ value \\
\hline$N$ & 566 & 212 & $|3|$ & 222 & \\
\hline Age & 50.98 & 50.16 & 56.5 & 48.56 & $\mathrm{p}=.000$ \\
\hline Weight (lbs) & 202.27 & 190.52 & 209.8 & 208.87 & $\mathrm{P}=.000$ \\
\hline Height (inches) & 63.76 & 63.15 & 64.2 & 64.07 & $\mathrm{p}=.00 \mathrm{I}$ \\
\hline Systolic BP (mmHg) & 128.04 & 125.27 & 132.54 & 127.98 & $\mathrm{p}=.000$ \\
\hline Diastolic BP (mmHg) & 80.31 & 80.83 & 80.32 & 80.66 & $\mathrm{P}=.529$ \\
\hline Hip (inches) & 46.99 & 46.24 & 48.38 & 47.2 & $\mathrm{P}=.006$ \\
\hline Waist (inches) & 42.4 & 41.6 & 43.45 & 42.77 & $\mathrm{P}=.006$ \\
\hline Mean Size of class & 17.65 & 16.47 & 16.9 & 18.82 & $\mathrm{p}=.000$ \\
\hline Sex (\%) & & & & & $\mathrm{P}=.173$ \\
\hline Female & 92.6 & 94.2 & 88.9 & 92 & \\
\hline Male & 7.4 & 5.8 & 11.1 & 8 & \\
\hline Race (\%) & & & & & $\mathrm{P}=.000$ \\
\hline Black/African-American & 48.5 & 42.4 & 70.4 & 48 & \\
\hline White & 22.7 & 21.2 & 11.1 & 32 & \\
\hline American Indian/Alaska Native & 1.2 & 0 & 0 & 4 & \\
\hline Asian/Pacific Islander & 0.6 & 1.2 & 0 & 0 & \\
\hline Multi-Racial & 4.3 & 5.9 & 0 & 4 & \\
\hline Other & 20.9 & 29.1 & 18.5 & 8 & \\
\hline Education (\%) & & & & & $\mathrm{p}=.120$ \\
\hline Elementary or Middle School & 1.9 & 2.4 & 3.7 & 0 & \\
\hline Some High School & 9.4 & 9.6 & 18.5 & 4 & \\
\hline High School or GED & 20.6 & 21.7 & 18.5 & 20 & \\
\hline Some College or Technical/Trade School & 51.3 & 53 & 44.4 & 52 & \\
\hline 4 Year College Degree or more & 16.9 & 13.3 & 14.8 & 24 & \\
\hline
\end{tabular}

\section{Program completion}

Approximately 53\% had no post-assessment data, yielding 251 participants in our primary analysis. Program completion between groups differed significantly, with Faith Center based groups showing the greatest percentage of participants with post-assessment data $(53 \%)$ and Health Center based groups with the lowest percentage $(29 \%)(\mathrm{Chi}=21.30, \mathrm{p}=0.000)$. Community Center based groups had a $50 \%$ completion rate. Among all groups, those that did not complete the program were more likely to weigh more at baseline (204.84 vs. $193.29 \mathrm{lbs}$ ), be Black/African American (63\% vs. 49\%) and have lower education.

\section{Weight change}

There was no significant difference in the weight change between the three groups using mean weight loss in pounds $(\mathrm{p}=0.058)$, mean weight loss percent $(\mathrm{p}=0.059)$ and by category of weight loss percent
$<5 \%$ or $\geq 5 \%$ ( $\mathrm{p}=0.391)$ with very few actually losing $\geq 5 \%$ of their initial body weight (Figure 1). After adjusting for sex, class size, race, baseline BMI, and education, we found a significant difference in mean weight loss in pounds by type of location $(p=0$.002). Similarly, after adjusting for confounders, the mean weight loss percent was also significant $(\mathrm{p}=0.001)$. We used a Bonferroni Adjustment to correct for multiple comparisons which indicated that the only significant difference for weight loss was Health Centers, whose participants lost significantly more pounds compared with Community Center based groups and lost significantly higher percentage of body weight compared with Faith Center groups. Adjusting for confounders did not significantly change the difference in category of weight loss percent. Using the intention to treat approach weight loss percent remained statistically significant among different groups $(\mathrm{p}=0.005)$ and weight loss in pounds became non-significant $(\mathrm{p}=0.059)$ (Table $2 \& 3)$. The difference in category of weight loss percent among location types remained non-significant (Chi $3.359 \mathrm{p}=0.187$ ). 
Table 2 Mean values for change in biometric measurements among program completers

\begin{tabular}{|c|c|c|c|}
\hline & Faith centers & Health centers & Community centers \\
\hline Mean Weight pre & $188.38(n=208)$ & $209.27(n=123)$ & $212.03(n=208)$ \\
\hline Mean Weight post & $183.61(n=111)$ & $192.70(n=38)$ & $212.15(n=112)$ \\
\hline \multirow{2}{*}{ Mean Weight Loss (lbs) } & $2.82(n=109)$ & $3.58(n=38)$ & $1.25(n=109)$ \\
\hline & [range -19 to 36 ] & [range -13 to 40.8 ] & [range -10 to 14.6$]$ \\
\hline Mean percentage weight loss & $1.44 \%(n=109)$ & $1.79 \%(n=38)$ & $0.58(n=109)$ \\
\hline Mean waist decrease (inches) & $1.41(n=109)$ & $1.70(n=37)$ & $0.38(n=108)$ \\
\hline Mean hip decrease (inches) & $0.95(n=104)$ & $0.91(n=37)$ & $-0.02(n=108)$ \\
\hline Mean decrease in Systolic Blood Pressure (mmHG) & $1.43(n=106)$ & $2.84(n=38)$ & $2.87(n=105)$ \\
\hline Mean decrease in Diastolic Blood Pressure (mmHG) & $1.05(n=106)$ & $-0.08(n=38)$ & $0.39(n=105)$ \\
\hline
\end{tabular}

Table 3 Mean changes in weight using imputed data

\begin{tabular}{llll}
\hline & Faith centers & Health centers & Community centers \\
\hline Mean Weight post & $186.74(n=2 \mid 2)$ & $208.16(n=131)$ & $211.21(n=223)$ \\
Mean Weight Loss (lbs) & $1.45(n=2 \mid 2)$ & $1.04(n=13 I)$ & $1.40(n=223)$ \\
Mean percentage weight loss & $0.74(n=2 \mid 2)$ & $0.50(n=131)$ & $0.72(n=223)$
\end{tabular}

\section{Weight Loss Percent by Location}

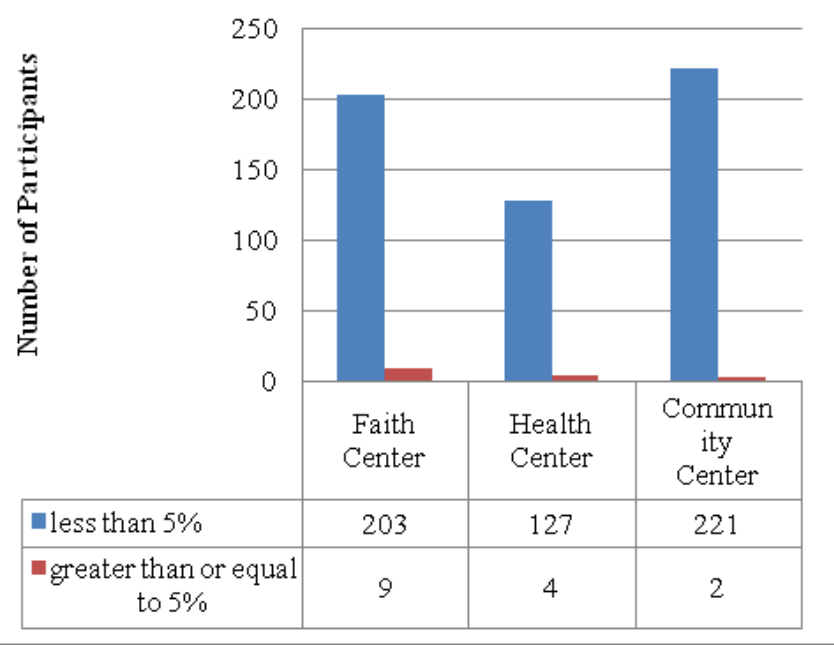

Figure I Category of weight loss percent by type of location.

\section{Waist and hip circumference}

We found a significant difference between groups with waist circumference change $(\mathrm{p}=0.008)$. Community Center based groups had significantly smaller decrease in waist circumference as compared to both Faith and Health Center based groups. After controlling for confounders, this difference was no longer significant $(p=0.059)$. Similarly, Community Center based participants experienced a small mean increase in their hip circumference compared to Faith and Health Center based groups, however this did not reach statistical significance ( $p=0.087, p=0.505$ after adjusting for confounders). In the intention to treat analysis, there was a significant difference in waist circumference change among groups, with Community Center based groups having the smallest change $(\mathrm{p}=0.026)$. Hip circumference change remained statistically non-significant $(\mathrm{p}=0.179)$.

\section{Blood pressure}

There were no significant differences between groups in change in Systolic Blood Pressure ( $\mathrm{p}=0.753$, after controlling for confounders $\mathrm{p}=0.787$ ) or Diastolic Blood pressure ( $\mathrm{p}=0.477$, after controlling for confounders $\mathrm{p}=0.998$ ). The intention to treat analysis was similar with non-significant differences for change in Systolic Blood Pressure $(p=0.672)$ and Diastolic Blood Pressure $(p=0.974)$.

\section{Discussion}

Among urban underserved participants in a Healthy Living Program, there was a small, but significant difference in the weight loss and percentage weight loss of those who participated in a Health Center based HLP compared with participants in Faith and Community Center based HLPs. In this population, we found the highest rates of program completion in Faith Based Center HLP's.

Our primary analysis shows a difference in weight loss related to the location of an intervention. Specifically, more pounds lost and greater percentage change from baseline weight among those who completed a Health Center based intervention. This is consistent with studies indicating that behavioral counseling by PCPs can influence patients' cardiovascular risk factors and behaviors. ${ }^{15}$ The participants in the Health Center based HLP are usually referred to the program by their PCP. The act of the PCP referral may have a similar influence as receiving behavioral counseling or may be done in conjunction with behavioral counseling. A recent meta-analysis of physician weight loss advice and patient weight loss attempts demonstrated a significant impact (OR 3.85); the authors also noted that there was a positive association between provider weight loss advice and actual patient 
weight loss. ${ }^{16}$ For HLP participants, we do not know the extent to which physician weight loss advice occurred along with the referral; this represents a potential confounder that could not be addressed in this study.

Physician referral to a behavioral change intervention may promote greater lifestyle change than a similar referral from a clergy-person or community member. In this study we cannot determine if our outcome is related to actual location in a health care center, or the efficacy of the program referral coming from a primary care physician. It is possible that PCP referrals to community center based interventions would result in the same effect. Future prospective studies could examine the impact of referral source on the success of behavioral change interventions.

The USPSTF recently recommended that primary care physicians screen all adults for obesity and offer or refer patients with a body mass index (BMI) of $30 \mathrm{~kg} / \mathrm{m}^{2}$ or higher to an intensive, multicomponent behavioral intervention program. ${ }^{17}$ This recommendation has been met with frustration by some primary care doctors about the paucity and expense of available intervention programs. ${ }^{18}$ Our study provides preliminary evidence suggesting that obesity interventions might work best if located at the primary care office, possibly through a group visit format which could improve access and be covered by health insurance.

\section{Limitations}

This analysis has several limitations, primarily due to different completion rates, making interpretation for some of the results a challenge. The intention to treat analysis resulted in a loss of significance, indicating a possible overestimate of the difference between groups in the initial analysis. The difference between the results of the original analysis and the intention to treat analysis is likely related to the high rates of drop-out, especially in health center based groups. It may also be related to the slight weight gain seen in the participants from the Community Center groups compared with an overall weight loss when using imputed data. Additionally, there were important baseline differences between the groups which we attempted to control for by adjusting for multiple potential confounders in the analysis. In a community setting, it is often not feasible or ethical to randomize individuals and this is a trade-off that community based "real-world" interventions frequently require.

Overall, our dropout rates were similar to what has been reported in the literature for community based prevention interventions, especially among low socio-economic status and racial/ethnic minority populations. ${ }^{19,20}$ We found was no difference in program completion based on referral by a PCP to the Health Center based programs. The Faith Centers had the highest rates of program completion with Health Centers having the lowest rates of completion. The HLP has been offered in Faith and Community Centers for a longer period of time than the Health Center based programs, so this may play a role in completion rates through improved familiarity or shared values in the communities they are trying to reach. The participants of Health Center based programs may also have been sicker or faced more barriers to participation. Previous studies have reported that poor eating behaviors, less physical activity, more depression, greater stress and higher emotional disturbance contribute to low retention among participants of weight loss interventions. ${ }^{21-23}$

Using completion of at least one variable of the post-assessment is an imperfect method of defining "Program Completion" as participants may have missed the last day when the assessment was completed or may have simply chosen not to complete the postassessment. Unfortunately, attendance was inconsistently tracked and therefore is not available for analysis. Further research with a larger number of participants is needed to look closer at the role of location in regards to retention and efficacy of interventions. Additionally, we are limited in the covariates that we can analyze to those collected as part of the original program. For example, no information about the leaders of the intervention is available. The group leader at a certain location may be the major influence, rather than some intrinsic value in the type of location. Additionally, the information may have been collected more thoroughly at certain locations as opposed to others, which could introduce bias. As mentioned earlier, participants were not randomly assigned to the location of their program, so the groups may differ in important aspects that we could not or did not measure.

\section{Strengths}

There are many strengths to this analysis. To our knowledge, comparing different types of locations with the same intervention has not been reported in the literature, making this a novel evaluation. Traditional interventions are often offered at multiple academic research institutions with a comparison to assure similar results at each institution. ${ }^{5}$ With the move in the implementation literature towards using a contextual view that the program location and delivery staff are equally important as the intervention and research team, this type of comparison will become even more relevant. Given the challenge of treating obesity and the immense need for accessible, effective interventions, these results could inform the expansion of group-based behavioral interventions for obesity to locations within Primary Care Offices or Health Centers.

\section{Conclusion}

A comprehensive behavioral modification intervention has been identified as the first line approach to treat the epidemic of obesity. Our analysis shows a small, but significant difference in the pounds lost and percentage weight loss of those who participated in a Health Center based HLP compared with those who participated in Faith and Community Center based HLPs. This study provides preliminary evidence that location may be an important element when designing and implementing interventions and that health center based interventions should be a focus of further obesity treatment research.

\section{Acknowledgements}

Funding: Work on this manuscript was supported by a grant from the National Institute of Mental Health (R25MH074898).

\section{Ethical approval}

This study protocol was reviewed by the RSRB at the University of Rochester Medical Center and deemed exempt.

\section{Conflict of interest}

The author declares no conflict of interest.

\section{References}

1. Oster G, Thompson D, Edelsberg J, et al. Lifetime health and economic benefits of weight loss among obese persons. Am J Public Health. 1999;89(10):1536-1542.

2. Wadden TA, Webb VL, Moran $\mathrm{CH}$, et al. Lifestyle modification for obesity: New developments in diet, physical activity, and behavior therapy. Circulation. 2012;125(9):1157-1170. 
3. Russell HA, Rufus C, Fogarty CT, et al. 'You need a support. when you don't have that ... chocolate looks real good'. barriers to and facilitators of behavioural changes among participants of a healthy living program. Fam Pract. 2013;30(4):452-458.

4. Rodgers GP, Collins FS. The next generation of obesity research: No time to waste. JAMA. 2012;308(11):1095-1096.

5. Glasgow RE, Chambers D. Developing robust, sustainable, implementation systems using rigorous, rapid and relevant science. Clin Transl Sci. 2012;5(1):48-55.

6. Glasgow RE, Vogt TM, Boles SM. Evaluating the public health impact of health promotion interventions: The RE-AIM framework. Am J Public Health. 1999;89(9):1322-1327.

7. Stange KC, Breslau ES, Dietrich AJ, et al. State-of-the-art and future directions in multilevel interventions across the cancer control continuum. J Natl Cancer Inst Monogr. 2012;2012(44):20-31.

8. Kramer MK, Kriska AM, Venditti EM, et al. Translating the diabetes prevention program: A comprehensive model for prevention training and program delivery. Am J Prev Med. 2009;37(6):505-511.

9. Lorig K, Sobel D, Laurent D. Living a healthy life with chronic conditions: Self-management of heart disease, arthritis, diabetes, asthma, bronchitis, emphysema \& others. 2nd ed. USA: Stanford University School of Medicine's Patient Education Research Center: Publishers Group West; 2000.

10. Wing RR, Lang W, Wadden TA, et al. Benefits of modest weight loss in improving cardiovascular risk factors in overweight and obese individuals with type 2 diabetes. Diabetes Care. 2011;34(7):1481-1486.

11. Lv J, Neal B, Ehteshami P, et al. Effects of intensive blood pressure lowering on cardiovascular and renal outcomes: A systematic review and meta-analysis. PLoS Med. 2012;9(8):e1001293.

12. Han TS, Richmond P, Avenell A, et al. Waist circumference reduction and cardiovascular benefits during weight loss in women. Int $J$ Obes Relat Metab Disord. 1997;21(2):127-134.
13. Janssen I, Katzmarzyk PT, Ross R. Waist circumference and not body mass index explains obesity-related health risk. Am J Clin Nutr. 2004;79(3):379-384.

14. Wadden TA, Neiberg RH, Wing RR, et al. Four-year weight losses in the look AHEAD study: Factors associated with long-term success. Obesity (Silver Spring). 2011;19(10):1987-1998.

15. Lin JS, O'Connor E, Whitlock EP, et al. Behavioral counseling to promote physical activity and a healthful diet to prevent cardiovascular disease in adults: A systematic review for the U.S. preventive services task force. Ann Intern Med. 2010;153(11):736-750.

16. Rose SA, Poynter PS, Anderson JW, et al. Physician weight loss advice and patient weight loss behavior change: A literature review and metaanalysis of survey data. Int J Obes (Lond). 2012;37(1):118-128.

17. Moyer VA, US Preventive Services Task Force. Screening for and management of obesity in adults: Clinical summary of U.S. preventive services task force. Ann Intern Med. 2012;157(5):373-378.

18. Brown M. AAFP, USPSTF recommend screening all adults for obesity, offering some patients lifestyle intervention. 2012.

19. Blumenthal DS, Sung J, Coates R, et al. Recruitment and retention of subjects for a longitudinal cancer prevention study in an inner-city black community. Health Serv Res. 1995;30(1 Pt 2):197-205.

20. Havas S, Anliker J, Greenberg D, et al. Final results of the maryland WIC food for life program. Prev Med. 2003;37(5):406-411.

21. Katzer L, Bradshaw AJ, Horwath CC, et al. Evaluation of a "nondieting" stress reduction program for overweight women: A randomized trial. Am J Health Promot. 2008;22(4):264-267.

22. Teixeira PJ, Going SB, Houtkooper LB, et al. Pretreatment predictors of attrition and successful weight management in women. Int J Obes Relat Metab Disord. 2004;28(9):1124-1133.

23. Yass-Reed EM, Barry NJ, Dacey CM. Examination of pretreatmen predictors of attrition in a VLCD and behavior therapy weight-loss program. Addict Behav. 1993;18(4):431-435. 